Supporting Information for

\title{
Construction of Tensile Strength and Density Prediction Models for Semi-Interpenetrating Polymer Network from Fluoroelastomer and Poly(dimethylsiloxane)
}

\author{
Ru-Qiu Zheng, Jie Jin*, Zheng-Hong Luo* \\ Department of Chemical Engineering, School of Chemistry and Chemical \\ Engineering, State Key Laboratory of Metal Matrix Composites, Shanghai Jiao Tong \\ University, Shanghai 200240, P. R. China
}

Corresponding Author: J. Jin; E-mail: xiachenche@ sjtu.edu.cn

Corresponding Author: Z.H. Luo; E-mail: luozh@sjtu.edu.cn

Tel.: +86-21-54745602; Fax: +86-21-54745602 

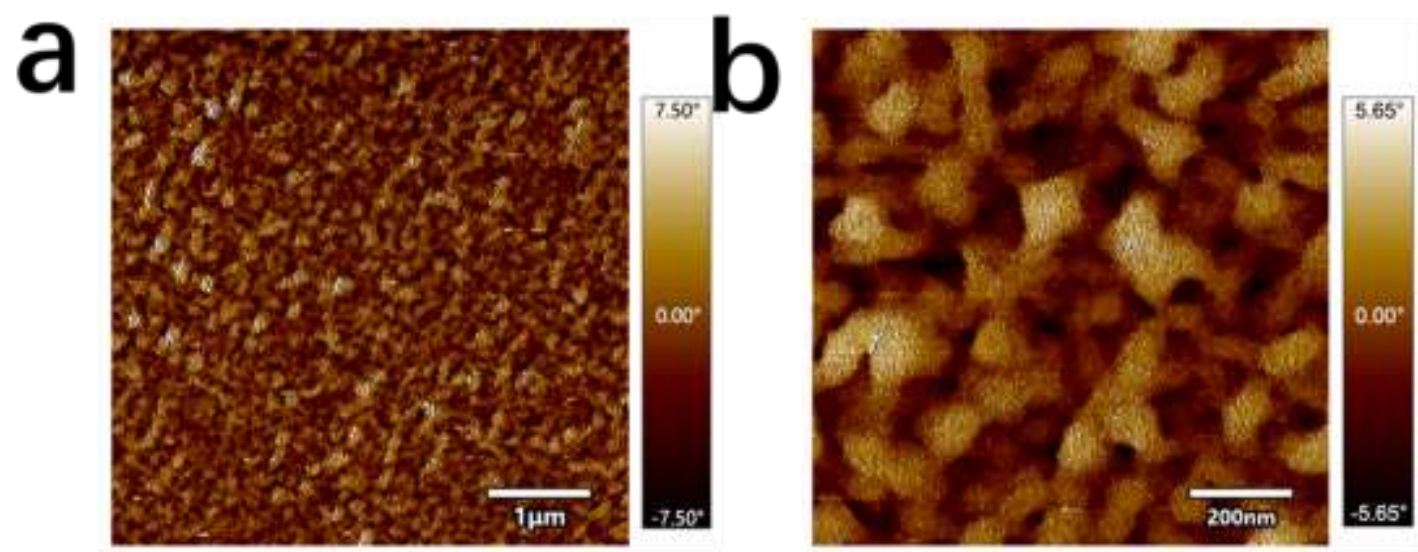

C
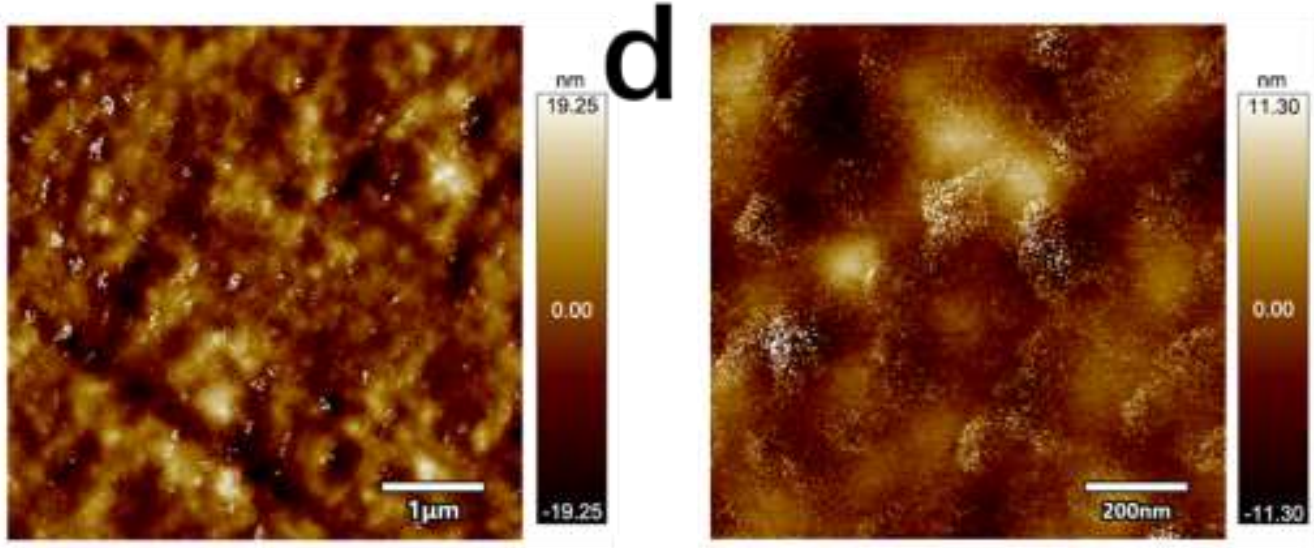

Figure S1. The AFM phase image and corresponding topographical image (a-c, b-d)

$$
\text { of semi-IPN }
$$
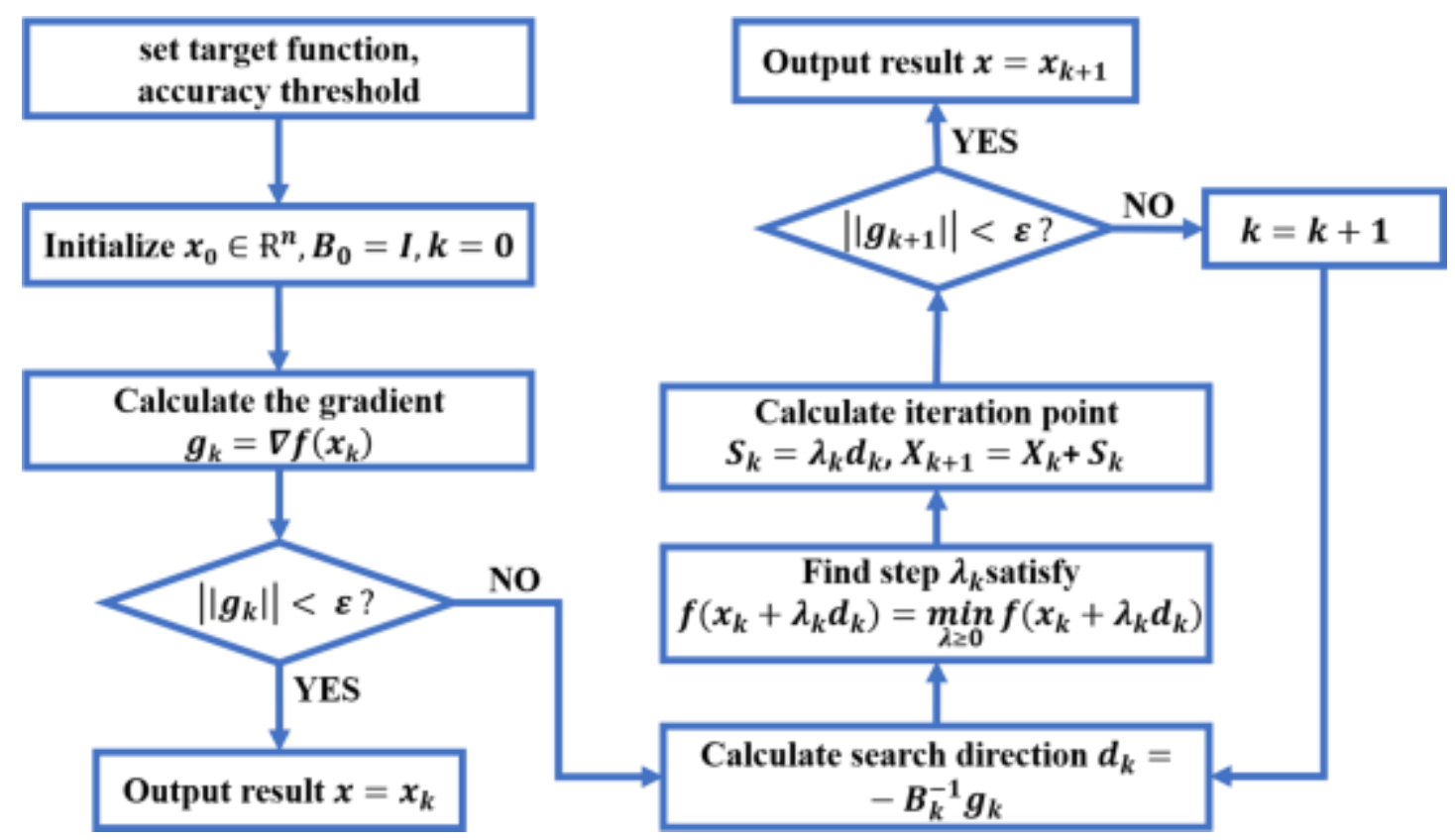

Figure S2. Flow chart of BFGS Quasi-Newton algorithm 
Table S1. Data of tensile strength used to calculate model parameters

\begin{tabular}{|c|c|c|c|c|c|}
\hline No. & $x_{1}$ & $x_{2}$ & {$[\mathrm{NCO}] /[\mathrm{OH}]$} & $M_{\mathrm{c}}(\mathrm{g} / \mathrm{mol})$ & $\sigma_{\exp }(\mathrm{Mpa})$ \\
\hline 1 & 0.60 & 0.40 & 1.00 & 6823.3 & 3.01 \\
\hline 2 & 0.60 & 0.40 & 1.00 & 6823.3 & 2.97 \\
\hline 3 & 0.60 & 0.40 & 1.00 & 6823.3 & 2.88 \\
\hline 4 & 0.70 & 0.30 & 1.00 & 6823.3 & 4.09 \\
\hline 5 & 0.70 & 0.30 & 1.00 & 6823.3 & 3.94 \\
\hline 6 & 0.70 & 0.30 & 1.00 & 6823.3 & 4.13 \\
\hline 7 & 0.80 & 0.20 & 1.00 & 6823.3 & 3.51 \\
\hline 8 & 0.80 & 0.20 & 1.00 & 6823.3 & 3.53 \\
\hline 9 & 0.80 & 0.20 & 1.00 & 6823.3 & 3.74 \\
\hline 10 & 0.70 & 0.30 & 0.98 & 7073.5 & 3.64 \\
\hline 11 & 0.70 & 0.30 & 0.98 & 7073.5 & 3.92 \\
\hline 12 & 0.70 & 0.30 & 0.98 & 7073.5 & 3.87 \\
\hline 13 & 0.70 & 0.30 & 1.04 & 7605.7 & 3.68 \\
\hline 14 & 0.70 & 0.30 & 1.04 & 7605.7 & 3.57 \\
\hline 15 & 0.70 & 0.30 & 1.04 & 7605.7 & 3.48 \\
\hline 16 & 0.70 & 0.30 & 1.06 & 7786.6 & 3.42 \\
\hline 17 & 0.70 & 0.30 & 1.06 & 7786.6 & 3.62 \\
\hline 18 & 0.70 & 0.30 & 1.06 & 7786.6 & 3.58 \\
\hline 19 & 0.70 & 0.30 & 1.10 & 8248.4 & 3.31 \\
\hline 20 & 0.70 & 0.30 & 1.10 & 8248.4 & 3.24 \\
\hline
\end{tabular}




\begin{tabular}{|c|c|c|c|c|c|}
\hline 21 & 0.70 & 0.30 & 1.10 & 8248.4 & 3.49 \\
\hline 22 & 0.65 & 0.35 & 1.00 & 6823.3 & 3.81 \\
\hline 23 & 0.65 & 0.35 & 1.00 & 6823.3 & 3.78 \\
\hline 24 & 0.65 & 0.35 & 1.00 & 6823.3 & 3.69 \\
\hline 25 & 0.75 & 0.25 & 1.00 & 6823.3 & 3.74 \\
\hline 26 & 0.75 & 0.25 & 1.00 & 6823.3 & 3.59 \\
\hline 27 & 0.75 & 0.25 & 1.00 & 6823.3 & 3.83 \\
\hline 28 & 0.70 & 0.30 & 1.02 & 7150.4 & 3.86 \\
\hline 29 & 0.70 & 0.30 & 1.02 & 7150.4 & 3.71 \\
\hline 30 & 0.70 & 0.30 & 1.02 & 7150.4 & 3.67 \\
\hline 31 & 0.70 & 0.30 & 1.08 & 8071.2 & 3.59 \\
\hline 32 & 0.70 & 0.30 & 1.08 & 8071.2 & 3.42 \\
\hline 33 & 0.70 & 0.30 & 1.08 & 8071.2 & 3.32 \\
\hline
\end{tabular}

Table S2. Data of density used to calculate model parameters

\begin{tabular}{cccc}
\hline No. & $x_{1}$ & $x_{2}$ & $\rho_{\exp }\left(\mathrm{g} / \mathrm{cm}^{3}\right)$ \\
\hline 1 & 0.60 & 0.40 & 1.3893 \\
2 & 0.60 & 0.40 & 1.3920 \\
3 & 0.70 & 0.30 & 1.4740 \\
4 & 0.70 & 0.30 & 1.4804 \\
5 & 0.70 & 0.30 & 1.4726 \\
6 & 0.70 & 0.30 & 1.4789
\end{tabular}




\begin{tabular}{cccc}
7 & 0.80 & 0.20 & 1.6153 \\
8 & 0.80 & 0.20 & 1.6248 \\
9 & 0.65 & 0.35 & 1.4364 \\
10 & 0.65 & 0.35 & 1.4174 \\
11 & 0.75 & 0.25 & 1.5286 \\
12 & 0.75 & 0.25 & 1.5158 \\
13 & 0.70 & 0.30 & 1.4855 \\
14 & 0.70 & 0.30 & 1.4813 \\
15 & 0.70 & 0.30 & 1.4819 \\
\hline
\end{tabular}

